Role of Intergrowths in the Properties of the Naturally Layered Manganites
A. Berger, R.M. Osgood III, J.S. Jiang, D.J. Miller,
Materials Science Division
Argonne National Laboratory, Argonne, IL 60439
The submitted manuscript has been created by the University of Chicago as Operator of Argonne National Laboratory ("Argonne") under Contract No. W-31-109-ENG-38 with the U.S. Department of Energy. The U.S. Government retains for itself, and others act- ing on its behalf, a paid-up, nonexclusive, irrevocable worldwide license in said article to reproduce, prepare derivative works, dis- tribute copies to the public, and perform pub- licly and display publicly, by or on behalf of the Government.

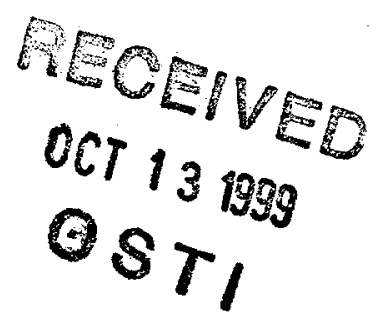

Proceedings of the $7^{\text {th }}$ NEC Symposium of Fundamental Approaches to New Material Phases, October 11-15, 1998, Nasu, Japan

Work supported by the U.S. Department of Energy Division of Basic Energy Sciences-Material Sciences under contract \#W-31-109-ENG-38. 


\section{DISCLAIMER}

This report was prepared as an account of work sponsored by an agency of the United States Government. Neither the United States Government nor any agency thereof, nor any of their employees, make any warranty, express or implied, or assumes any legal liability or responsibility for the accuracy, completeness, or usefulness of any information, apparatus, product, or process disclosed, or represents that its use would not infringe privately owned rights. Reference herein to any specific commercial product, process, or service by trade name, trademark, manufacturer, or otherwise does not necessarily constitute or imply its endorsement, recommendation, or favoring by the United States Government or any agency thereof. The views and opinions of authors expressed herein do not necessarily state or reflect those of the United States Government or any agency thereof. 


\section{DISCLAIMER}

Portions of this document may be illegible in electronic image products. Images are produced from the best available original document. 


\title{
Role of Intergrowths in the Properties of the Naturally Layered Manganites
}

\author{
A. Berger, R. M. Osgood III, J. S. Jiang, D. J. Miller, J. F. Mitchell, \\ and S. D. Bader \\ Materials Science Division, \\ Argonne National Laboratory, Argonne, IL 60439 (USA)
}

\begin{abstract}
:
The structural and magnetic properties of the two-layered RuddlesdenPopper phase $\mathrm{SrO}\left(\mathrm{La}_{1-\mathrm{x}} \mathrm{Sr}_{\mathrm{x}} \mathrm{MnO}_{3}\right)_{2}$ with $\mathrm{x}=0.3$ and $\mathrm{x}=0.4$ are investigated. These naturally layered manganites exhibit a colossal magnetoresistance, a magnetic anisotropy which is strongly compositiondependent, almost no remanence, and a non-vanishing magnetization in a extended temperature range above the Curie temperature $\left(T_{C}\right)$. The magnetization in this temperature range is not intrinsic to the crystal, but is attributed to intergrowths. These two-dimensional lattice imperfections consist of additional or missing SrO layers and have been observed in transmission electron micrographs. The magnetic properties of the intergrowths differ from the bulk crystal which results in unusual magnetic behavior.
\end{abstract}

Keywords: intergrowth, colossal magnetoresistance, layered materials, manganites, magnetic measurements 


\section{Introduction:}

The renewed strong interest in doped manganites has been triggered by interest in the colossal magnetoresistance (CMR) effect [1]. Here, one observes a large reduction of the resistivity upon applying a magnetic field. The CMR effect is caused by the temperature dependent phase transition from a paramagentic insulator to a ferromagnetic metal in these doped manganites. Thus, in the vicinity of this phase transition an applied magnetic field does not only restore the magnetic order as it does in all types of ferromagnetic materials but also gives rise to metallic conductivity. The coincidence of the ferromagnetic-paramagnetic phase transition with the metal-insulator transition, that accompanies the CMR effect, is associated with the double exchange mechanism [2]. Due to the CMR effect, the ferromagnetic manganites are of technological interest and a vast amount of work has been conducted towards optimizing the CMR properties with respect to the resistivity change, the necessary magnetic fields, as well as the useful temperature range. In addition to these important technological aspects, the doped manganites are fascinating materials and exhibit a wide variety of unusual physical properties which are related to the magnetically ordered state and make these materials considerably more complex than conventional transition metal ferromagnets.

Most CMR studies have focused on the perovskite manganites like $\mathrm{La}_{1-\mathrm{x}} \mathrm{Sr}_{\mathrm{x}} \mathrm{MnO}_{3}$. However, Moritomo et al. demonstrated that it is possible to fabricate a layered form of these materials, in particular the $n=2$ variant of the Ruddlesden-Popper series $(\mathrm{La}, \mathrm{Sr})_{\mathrm{n}+1} \mathrm{Mn}_{\mathrm{n}} \mathrm{O}_{3 \mathrm{n}+1}$ [3]. Figure 1 illustrates the $n=1,2, \infty$ members of this family. The unit cell may be written as $\mathrm{SrO}\left(\mathrm{La}_{1-\mathrm{x}} \mathrm{Sr}_{\mathrm{x}} \mathrm{MnO}_{3}\right)_{\mathrm{n}}$ with $n$ being the number of $\mathrm{MnO}_{6}$ octahedral layers in each unit cell. The $n=\infty$ compound then refers to the 
bulk perovskite structure, whereas the $n=1$ member consists of separated $\mathrm{MnO}_{6}$ layers and does not show ferromagnetic order at accessible temperatures. The bilayer structure $n=2$, however, has interesting magnetic and related properties and is presently the focus of intense research efforts [3-13]. The unit cell consists of two $\mathrm{MnO}_{6}$ octahedral layers which are separated by an insulating $\mathrm{SrO}$ layer from the adjacent bilayer. The structure is tetragonal, with the ab-plane parallel to the layers of $\mathrm{MnO}_{6}$ [4]. The $\mathrm{x}=0.4$ variant of this material was reported to have a $20,000 \%$ CMR with $\mathrm{H}=7 \mathrm{~T}$, and $\sim 200 \% \mathrm{CMR}$ at low fields $(0.3 \mathrm{~T})$ for $\mathrm{T}$ $=129 \mathrm{~K}$ [3]. All of these materials (including the $n=\infty$ variety) exhibit a competition between antiferromagnetism (due to superexchange) and ferromagnetism (due to double exchange). For $x=0$, the materials show antiferromagnetic order, while for $x \approx 0.2-0.4$, the materials are ferromagnetic, and exhibit a metal-insulator transition at $T_{c} \cdot T_{c}$ broadens and moves appreciably in an external field $\mathrm{H}$, which in turn shifts and broadens the metal-insulator transition and causes the CMR. An external field is thought to align the Mn spins and therefore allows the dopinginduced excess electrons (via the double exchange mechanism) to delocalize and "hop" between the $\mathrm{Mn}^{3+}$ and $\mathrm{Mn}^{4+}$ sites [2].

Besides the CMR effect the bilayer phase $\mathrm{SrO}\left(\mathrm{La}_{1-\mathrm{x}} \mathrm{Sr}_{\mathbf{x}} \mathrm{MnO}_{3}\right)_{2}$ exhibits a number of interesting structural and magnetic properties. The structural properties have been extensively investigated using X-ray and neutron scattering and unusually large magnetoelastic effects have been observed [7]. Furthermore, the observed magnetic order strongly depends on the exact value of $x$ and shows a rich variety of ordered states [11]. For the $\mathrm{x}=0.3$ and $\mathrm{x}=0.4$ compositions, a ferromagnetic ground state is observed with the easy axis of the magnetization along the c-axis and in the ab-plane, respectively. Both types of materials exhibit a strong CMR effect 
and a very anisotropic conductivity below as well as above $T_{c}$ due to the insulating SrO planes that separate the bilayers [11]. However, a quantitative understanding of the observed properties is still missing. Also, the critical behavior and the magnetic correlations near $T_{c}$ have been studied but a clear understanding of all aspects of the ferromagnetically ordered state is presently not available [13]. More studies are necessary to explore the rich variety of phenomena found in these layered materials. In particular, the role of polarons in governing the energetics of the phase transition needs further elucidation.

As in the cuprate superconductors, not all of the interesting physics for the manganites is confined in the temperature region $T<T_{C}$ [14]. Several studies have reported short-range magnetic order for $T>T_{C}$ in these layered materials (both $\mathrm{x}=0.3$ and 0.4) $[4,10,15]$. Besides the possibility of unusual intrinsic effects, due to the complexity of the materials, it has also been reported that these layered materials contain intergrowth defects [11]. Certain magnetic phenomena observed in those materials are caused or influenced by these extrinsic sample imperfections which can be envisioned as an occasional stacking fault with missing or added SrO spacer layers. Thus, an awareness of the presence of intergrowths and knowledge of their extent and influence is useful in understanding the layered CMR materials. This article focuses primarily on the intergrowths. After a brief discussion of experimental procedures in Sec. 2 we explore the structural properties of the intergrowths (Sec. 3). Magnetic and magnetoresistance properties are then analyzed in Sec. 4. Finally, we summarize our results in Sec. 5 and discuss the interest in intergrowth structures as atomically flat inclusions with two-dimensional physical properties. 


\section{EXPERIMENTAL}

Crystals of $\mathrm{La}_{2-2 x} \mathrm{Sr}_{1+2 x} \mathrm{Mn}_{2} \mathrm{O}_{7} \quad(\mathrm{x}=0.3,0.4)$ were grown from polycrystalline rods of the same nominal composition using the travelingfloating-zone technique in an optical image furnace (NEC model SCM15HD). The precursor rods were prepared by solid-state synthesis from high purity (>99.99\%) starting materials: $\mathrm{La}_{2} \mathrm{O}_{3}$ (prefired in flowing $\mathrm{O}_{2}$ at $1000^{\circ} \mathrm{C}$ for $12 \mathrm{hrs}$ ), $\mathrm{MnO}_{2}$, and $\mathrm{SrCO}_{3}$. After several firings at 1000 $1350^{\circ} \mathrm{C}$, the powders were isostatically pressed into rods suitable for zone melting. The growth atmosphere was $20 \% \mathrm{O}_{2}$. In each case, the crystals grew with the c-axis normal to the zone travel direction. The resulting highly textured polycrystalline boules can be cleaved readily to yield shiny black crystals of layered manganite. Typical dimensions of the cleaved crystals are $2 \times 2 \times 0.1 \mathrm{~mm}^{3}$. Back-reflection x-ray Laue photographs establish that in all cases the cleaved crystals have the $\mathrm{c}$-axis oriented normal to the thin plates. Detailed investigations of the atomic structure of these crystals using neutron diffraction show the good quality of our samples including the full occupancy of all lattice sites [7]. For the investigation of the microscopic structure, in particular the study of intergrowths, we have performed TEM measurements on ion-milled platelets. Magnetization and susceptibility $(\chi)$ measurements were made on both, a SQUID and an extraction magnetometer from Quantum Design, equipped with 7 and $9 \mathrm{~T}$ superconducting solenoids, respectively. The trapped flux in the solenoids $(\sim 10 \mathrm{Oe})$ was monitored, so that the field values given are correct to within $\pm 0.2 \mathrm{Oe}$. Measurements were made with the applied field both parallel to the ab-plane $(\mathrm{H} \| \mathrm{ab})$ and parallel to the c-axis $(\mathrm{H} \| \mathrm{c})$. Resistivity measurements were made on thin platelets obtained by crushing the boule; leads were connected by attaching In wires to sputtered Au contacts. 


\section{Intergrowth Structures}

Seshadri et al. have demonstrated the presence of intergrowths in these layered materials, using transmission electron microscopy (TEM) [9]. Intergrowths can be due to missing or extra layers of SrO atoms between the $\mathrm{MnO}_{6}$ octahedral planes. Such defects represent $n \neq 2$ variants of the Ruddlesden-Popper series. Figure 2 displays high-resolution TEM images from samples with $x=0.4$. The most prominent feature in these pictures are the white bands which form a regular array of horizontal lines. A detailed analysis reveals that these white bands are associated with the insulating $\mathrm{SrO}$ double layers which can be seen from the inset. So, the imperfections seen in Fig. 2 correspond to missing or extra SrO layers. In Fig. 2a, the regular series of white bands is interrupted by a $n=5$ intergrowth structure, whereas in Fig. 2b, an extra white band is present which divides the regular units cell into two $n=1$ intergrowth features. Such intergrowths are also observed in the $\mathrm{x}=0.3$ samples as can be seen from Fig. 3 where two bright-field micrographs are shown. Here, the detailed atomic structure of the intergrowths is not visible due to the lower resolution. However, the intergrowths can still be identified as sharp vertical lines, perpendicular to the c-axis of the crystal (Fig. 3b). In Fig. $3 \mathrm{a}$, no intergrowth is detectable, which means that the depicted segment of this crystal exhibits perfect $n=2$ bilayer periodicity.

The intergrowth structures influence the magnetic properties of $\mathrm{SrO}\left(\mathrm{La}_{1-\mathrm{x}} \mathrm{Sr}_{\mathrm{x}} \mathrm{MnO}_{3}\right)_{2}$ because they represent inclusions of a different variant of the Ruddleson-Popper series and therefore a different magnetic entity. As already mentioned, the magnetic properties of the layered materials depend strongly on $n$ and therefore it is reasonable to assume that the intergrowth features within the lattice also have magnetic properties 
different from the adjacent "perfect" layers. Thus, it is very important to characterize the magnetic properties of the intergrowth phase as well as its influence on the general behavior of the layered material. An estimate of the intergrowth volume fraction can be made from magnetization measurements. We obtain a volume fraction of $0.1-1 \%$ intergrowths in the $x=0.4$ samples and $0.3-0.6 \%$ intergrowths in the $x=0.3$ samples.

\section{Magnetic and Transport Properties}

Before we discuss the influence of the intergrowths on the magnetic and transport properties, it is necessary to analyze the basic properties of the $\mathrm{SrO}\left(\mathrm{La}_{1 \cdot \mathrm{x}} \mathrm{Sr}_{\mathrm{x}} \mathrm{MnO}_{3}\right)_{2}$ host material itself. Figure 4 shows the measured magnetic moment for an $\mathrm{x}=0.4$ sample in a relatively small applied field as a function of temperature. Here, we see the behavior of a typical ferromagnet, showing a substantial magnetic moment in a moderate external magnetic field up to $T_{C}$ where the ferromagnetic order breaks down. The observed $T_{C}$ 's are $120 \mathrm{~K}$ for $\mathrm{x}=0.4$ and $90 \mathrm{~K}$ for $\mathrm{x}=0.3$ samples. For $\mathrm{x}=0.4$, the ab-plane is the easy plane of the magnetization with a weak four-fold anisotropy, whereas samples with $x=0.3$ yield an out-of-plane orientation of the magnetization. The magnetocrystalline anisotropy is obvious from Fig. 5 where low temperature $\mathrm{M}(\mathrm{H})$ measurements of such samples are shown for different orientations of the externally applied field. The typical CMR behavior of these materials can be found in Fig. 6. Here, the temperature dependent resistivity for an $x=$ 0.3 sample is displayed with the current parallel (Fig. 6a) and perpendicular (Fig. 6b) to the ab-plane. The observed resistivity parallel to the ab-plane is two orders of magnitudes smaller than the c-axis resistivity, but the temperature and field dependence are alike in both cases. At $T_{C}$, one observes an abrupt drop of the resistivity for decreasing temperatures 
consistent with the paramagnetic-to-ferromagnetic phase transition. Upon application of a large magnetic field $(\mathrm{H}=9 \mathrm{~T}$ in our measurements), this phase transition is smeared out and a significant conductivity is restored above $T_{C}$ resulting in the $C M R$ effect.

Besides understanding the basic magnetic and resistance properties in terms of the dominant magnetic interactions, $\mathrm{SrO}\left(\mathrm{La}_{1-\mathrm{x}} \mathrm{Sr}_{\mathbf{x}} \mathrm{MnO}_{3}\right)_{2}$ provides another challenge associated with materials issues. As appears in Fig. 4, the magnetization shows a transition at $T_{c}$, but one can also see from these data that the measured magnetization does not vanish or even approach $M=0$ for $\mathrm{T}>\mathrm{T}_{\mathrm{C}}$. This can be seen in more detail in Fig. 7, where only the temperature range near $T_{c}$ is depicted. In Fig. $7 \mathrm{a}$, we see the measured magnetic moment as a function of temperature for several values of the externally applied field. The observed data for large fields are consistent with the expected behavior of a field induced magnetic moment even above $T_{C}$. This field induced moment should decrease with increasing temperature as one can see in the case of $\mathrm{H}=5 \mathrm{kOe}$. For low fields, however, the observed behavior is surprising because even for fields as low as $0.2 \mathrm{kOe}$ a significant magnetic moment is observed even $20 \mathrm{~K}$ above $T_{C}$. Furthermore, the moment seems to be almost independent of the temperature as one moves further away from $T_{C}$. This rather unusual behavior becomes even more evident in Fig. $7 \mathrm{~b}$ where we have plotted the inverse susceptibility vs. temperature. Here, one would expect the curves taken for various field strength to disagree only in the vicinity of $T_{C}$ but approach each other for higher temperatures. This is obviously not the case and therefore, the observed magnetization measurements cannot be interpreted in terms of a field induced magnetization above $T_{C}$. Instead, these results demonstrate the existence of a second magnetic entity with a higher ordering temperature in our samples. As already outlined in our 
discussion of the intergrowth structure, we would expect the intergrowth to have different magnetic properties and it seems reasonable to assume that an $n>2$ intergrowth structure should have an increased ordering temperature. Thus, we can associate the observed second magnetic entity in our samples with structural intergrowths. An additional argument for this explanation is the fact that the residual magnetization above $T_{C}$, as a fraction of the total magnetic moment of the entire sample, varies from specimen to specimen, clearly indicating an extrinsic origin for the residual magnetization. We should also keep in mind that we saw such a strong variation for the density of intergrowth structures in Fig. 3.

To establish the connection between the residual magnetization and the intergrowths, we have also studied the residual magnetization in more detail. Figure 8 shows the observed magnetic moment as a function of temperature for an $\mathrm{x}=0.3$ sample. As we can see, even at room temperature, i. e. at temperature values three times as large as the $T_{C}$ of the host material, we still find a significant magnetic moment with only a very small magnetic field applied. In addition, we observe that the $\mathrm{M}(\mathrm{T})$ behavior shows three individual transition-like features. This highly unusual temperature dependence indicates that there are at least three different types of additional phases in this sample. This is in agreement with our interpretation because one would expect a variety of intergrowth structures with different $n$-values or spacial extents to be present in our samples. Figure 9 shows the field dependence of the magnetization for the $\mathrm{x}=0.3$ sample at $\mathrm{T}=250 \mathrm{~K}$ for in-plane as well as out-of-plane orientation of the applied field. Interestingly, one finds the residual magnetization to be oriented within the ab-plane as opposed to an orientation along the caxis found below $T_{c}$ for this very sample. Thus, the preferred magnetization orientation changes at $T_{C}$, which can be explained within our 
picture of intergrowth magnetism. Below $T_{C}$, the entire sample is ferromagnetic and the magnetization prefers the orientation along the magnetocrystalline easy axis. Above $T_{C}$, however, the ferromagnetic phase is present only in a thin layer, namely the intergrowth phase, which exhibits a strong shape anisotropy forcing the magnetization to lie within the ab-plane. Thus, the association of the residual magnetization with the observed structural intergrowth features gives a natural explanation for the reorientation of the magnetization above $T_{C}$.

\section{Summary and Outlook}

The two-layered Ruddlesden-Popper phase $\mathrm{SrO}\left(\mathrm{La}_{1-\mathrm{x}} \mathrm{Sr}_{\mathrm{x}} \mathrm{MnO}_{3}\right)_{2}$, with $\mathrm{x}=$ 0.3 and $x=0.4$, exhibits CMR near $T_{C}$ and a magnetic anisotropy which is strongly composition-dependent. The magnetic order observed for $T>T_{C}$ includes not only the field induced or short-range order response of the intrinsic material, but a ferromagnetic signal due to structural imperfections known as intergrowths. These intergrowths are twodimensional lattice defects and are visible in TEM images with an orientation parallel to the ab-planes. Due to their planar structure, the intergrowths exhibit a magnetization orientation within the ab-plane even in the $\mathrm{x}=0.3$ material, despite the fact that the c-axis is the magnetocrystalline easy axis for this composition. The $x=0.3$ and 0.4 compositions differ strongly only in the magnetic anisotropy of the intrinsic material, not in the temperature dependence of the resistivity or magnetization.

The intergrowth structures and the associated magnetism are significant in two aspects. First, they are essentially isolated magnetic entities for temperatures above $T_{c}$ and allow investigations of twodimensional ferromagnetism in a system with a non-conventional exchange 
mechanism. They are also an example of a two-dimensional ferromagnetic material buried inside a host material with exchange enhanced magnetic susceptibility, which makes them an interesting research topic. Secondly, the intergrowth magnetism plays a role for the magnetic properties of the host material in the vicinity of $\mathrm{T}_{\mathrm{c}}$. Here, the layered $\mathrm{SrO}\left(\mathrm{La}_{1-\mathrm{x}} \mathrm{Sr}_{\mathrm{x}} \mathrm{MnO}_{3}\right)_{2}$ is not a simple bulk phase near its ordering temperature, but rather a binary system of a majority phase coupled to a ferromagnetic minority phase. Thus, the magnetic and resistivity properties are complex and challenging.

\section{Acknowledgments}

This work has been supported by the U. S. Department of Energy, Basic Energy Sciences - Materials Sciences under Contract W-31-109-ENG-38. 


\section{References}

' R. van Helmolt, J. Wecker, B. Holzapfel, L. Schultz, and K. Samwer, Phys. Rev. Lett. 71, 2331 (1993).

${ }^{2}$ C. Zener, Phys. Rev. 82403 (1951).

${ }^{3}$ Y. Moritomo, A. Asamitsu, H. Kuwahara, and Y. Tokura, Nature 380 141 (1996).

${ }^{4}$ T. Kimura, Y. Tomioka, H. Kuwahara, A. Asamitsu, M. Tamura, and Y. Tokura, Science 2741698 (1996).

${ }^{5}$ P. D. Battle, M. A. Green, N. S. Laskey, J. E. Millburn, P. G. Radaelli, M. J. Rosseinsky, S. P. Sullivan, and J. F. Vente, Phys. Rev. B 5415967 (1996).

${ }^{6}$ P. Laffez, G, Van Tendeloo, R. Seshadri, M. Hervieu, C. Martin, A. Maignan, and B. Raveau, J. Appl. Phys. 805850 (1996).

7 J. F. Mitchell, D. N. Argyriou, J. D. Jorgensen, D. G. Hinks, C. D. Potter, and S. D. Bader, Phys. Rev. B 5563 (1997).

${ }^{8}$ D. N. Argyriou, J. F. Mitchell, J. B. Goodenough, O. Chmaissem, S. Short, J. D. Jorgensen, Phys. Rev. Lett. 781568 (1997).

${ }^{9}$ R. Seshadri, M. Hervieu, C. Martin, A. Maignan, B. Domenges, B. Raveau, and A. N. Fitch, Chem. Mater. 91778 (1997). 
${ }^{10}$ C. D. Potter, Maribeth Swiatek, S. D. Bader, D. N. Argyriou, J. F. Mitchell, D. J. Miller, D. G. Hinks, and J. D. Jorgensen, Phys. Rev. B 57 72 (1998)

${ }^{11}$ S. D. Bader, R. M. Osgood III, D. J. Miller, J. F. Mitchell, and J. S. Jiang, J. Apply. Phys. 836385 (1998).

${ }^{12}$ S. Rosenkranz, R. Osborn, J. F. Mitchell, L. Vasiliu-Doloc, J. W. Lynn, S. K. Sinha, and D. N. Argyriou, J. Apply. Phys. 837348 (1998).

${ }^{13}$ R. Osborn, S. Rosenkranz, D. N. Argyriou, L. Vasiliu-Doloc, J. W. Lynn, S. K. Sinha, J. F. Mitchell, K. E. Gray, and S. D. Bader, Phys. Rev. Lett. (in press)

${ }^{14}$ H. Ding, T. Yokoya, J. C. Campuzano, T. Takahashi, M. Randeria, M. R. Norman, T. Mochiku, K. Hadowaki, J. Giapintzakis, Nature 38251 (1996).

${ }^{15}$ T. G. Perring, G. Aeppli, Y. Moritomo, and Y. Tokura, Phys. Rev. Lett. 783197 (1997). 


\section{Figure Captions}

Fig. 1: Diagram of the structure $\mathrm{SrO}\left(\mathrm{La}_{1-x} \mathrm{Sr}_{\mathbf{x}} \mathrm{MnO}_{3}\right)_{\mathrm{n}}$ : the variable $n$ refers to the number of $\mathrm{MnO}_{6}$ octahedral layers in the structure. The atoms shown are the $\mathrm{La}, \mathrm{Sr}$ cations in between the $\mathrm{O}_{6}$ octahedra which surround the $\mathrm{Mn}$ atoms.

Fig. 2: High-resolution TEM micrograph showing the detailed intergrowth structure (marked with a pointer) for the $\mathrm{x}=0.4$ composition: (a) intergrowth with $n=5$, (b) intergrowth structure corresponding to two $n=$ 1 unit cells.

Fig. 3: Bright-field TEM micrograph showing (a) perfect crystal (b) crystal with intergrowths. In (b) the intergrowths are visible as vertical streaks in the photograph (marked with pointers).

Fig. 4: Magnetic moment $v s$. temperature for a $\mathrm{SrO}\left(\mathrm{La}_{1-x} \mathrm{Sr}_{\mathrm{x}} \mathrm{MnO}_{3}\right)_{2}$ sample with $\mathrm{x}=0.4(\mathrm{H}=0.5 \mathrm{kOe} \| \mathrm{ab})$.

Fig. 5: Magnetization (normalized to the saturation magnetization $\mathrm{M}_{\mathrm{s}}$ ) $v s$. applied field $\mathrm{H}$ for (a) $\mathrm{x}=0.4$ (b) $\mathrm{x}=0.3$ ( $\mathrm{H} \|$ ab: filled symbols, $\mathrm{H} \| \mathrm{c}$ : clear symbols, the temperature was held constant at $\mathrm{T}=5 \mathrm{~K}$ in all measurements).

Fig. 6: Resistivity vs. temperature for $\mathrm{SrO}\left(\mathrm{La}_{1-x} \mathrm{Sr}_{\mathrm{x}} \mathrm{MnO}_{3}\right)_{2}(\mathrm{x}=0.3)$ in zero field and at $\mathrm{H}=9 \mathrm{~T}$ for (a) $\mathrm{j} \| \mathrm{ab}$, and (b) $\mathrm{j} \| \mathrm{c}$. The resulting magnetoresistance values are shown (thick line) on the right hand side. 
Measurements were made on separate samples, both of whom had $T_{C} \approx 90$ $\mathrm{K}$; the minor difference between the $\mathrm{T}_{\mathrm{C}}$ 's was normalized out.

Fig. 7: Magnetic moment vs. temperature of a $\mathrm{SrO}\left(\mathrm{La}_{1-\mathrm{x}} \mathrm{Sr}_{\mathrm{x}} \mathrm{MnO}_{3}\right)_{2}(\mathrm{x}=$ 0.4 ) sample measured for a certain number of field values $H$ : (a) original data, (b) inverse susceptibility $\chi^{-1}$ values deduced from the data.

Fig. 8: Magnetic moment vs. temperature for a $\mathrm{SrO}\left(\mathrm{La}_{1-x} \mathrm{Sr}_{\mathrm{x}} \mathrm{MnO}_{3}\right)_{2}$ sample with $x=0.3(H=15 \mathrm{Oe} \| \mathrm{ab})$. Three different transitions at $T_{i}^{*}$ are identified.

Fig. 9: Magnetic moment vs. applied field for a $\mathrm{SrO}\left(\mathrm{La}_{1-\mathrm{x}} \mathrm{Sr}_{x} \mathrm{MnO}_{3}\right)_{2}$ sample ( $\mathrm{x}=0.3$ ) at $\mathrm{T}=250 \mathrm{~K}$ with $\mathrm{H} \| \mathrm{ab}$ (filled symbols) and $\mathrm{H} \| \mathrm{c}$ (clear symbols). Both measurements are superimposed on a linear paramagnetic background from the intrinsic portion of the crystal. 


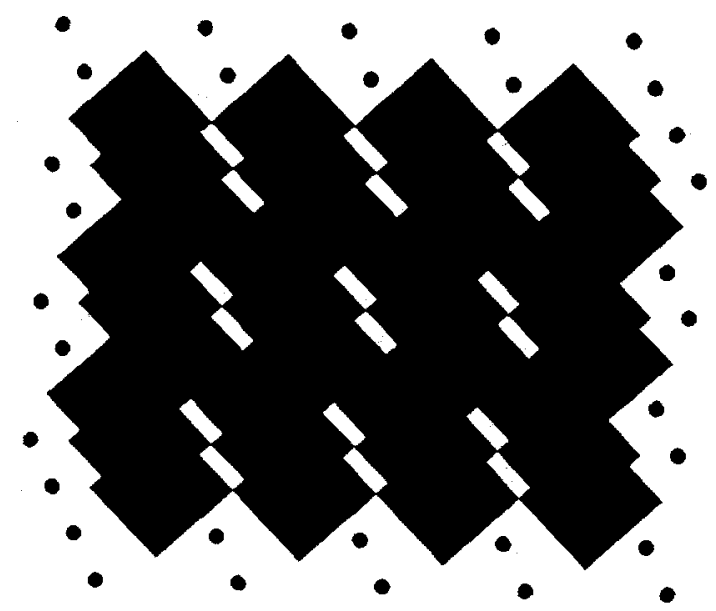

8

II
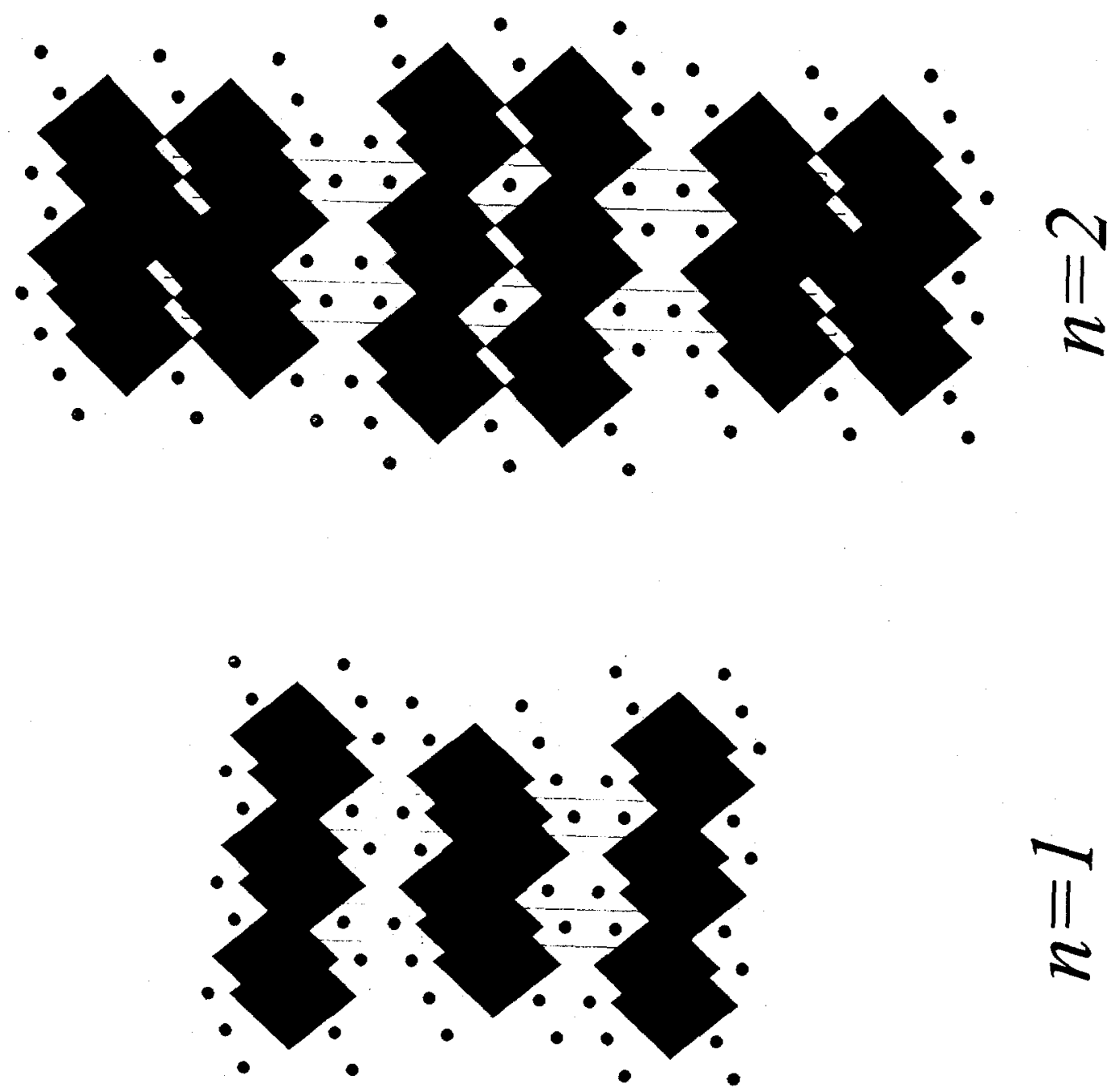

$N$ 


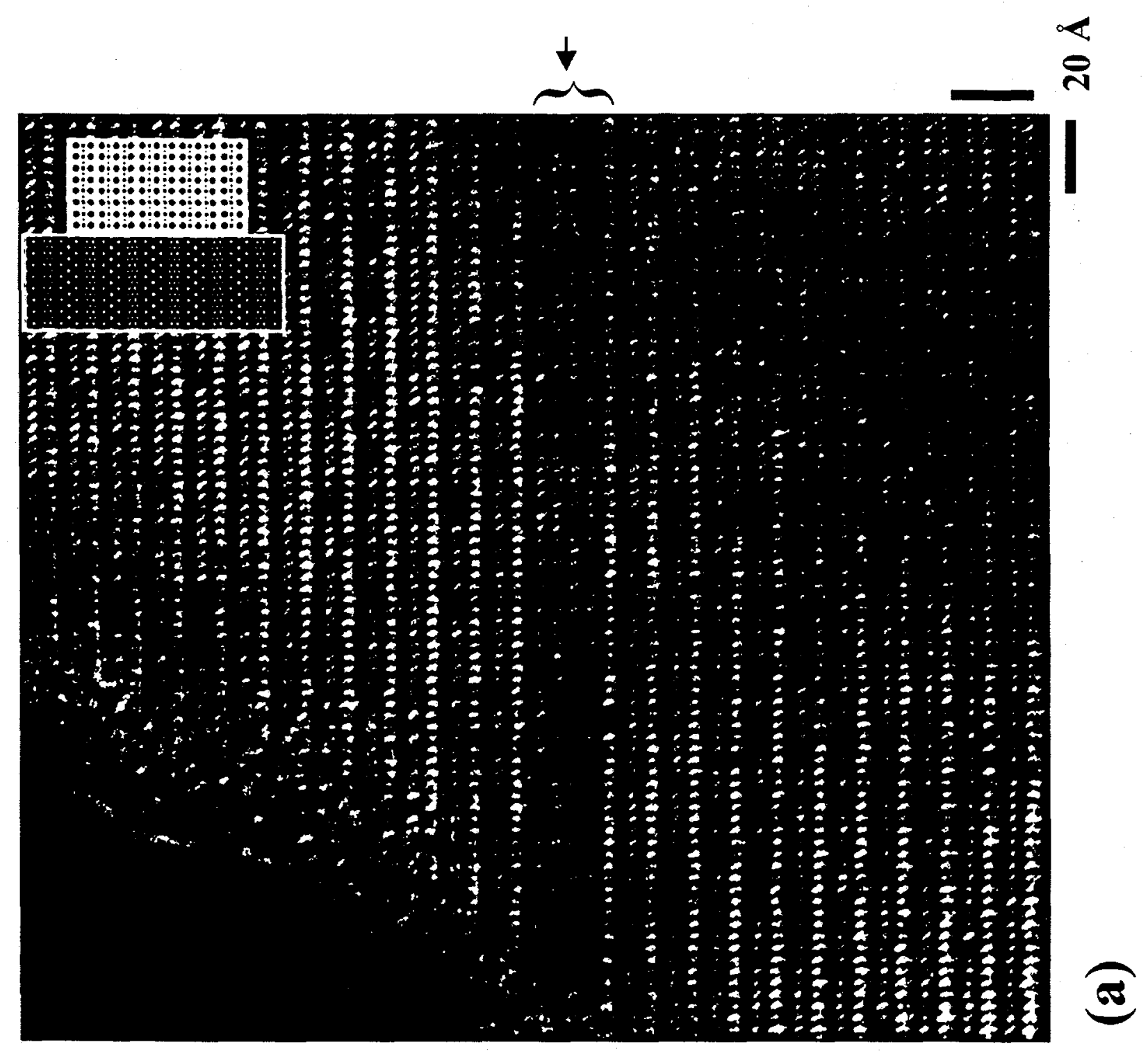




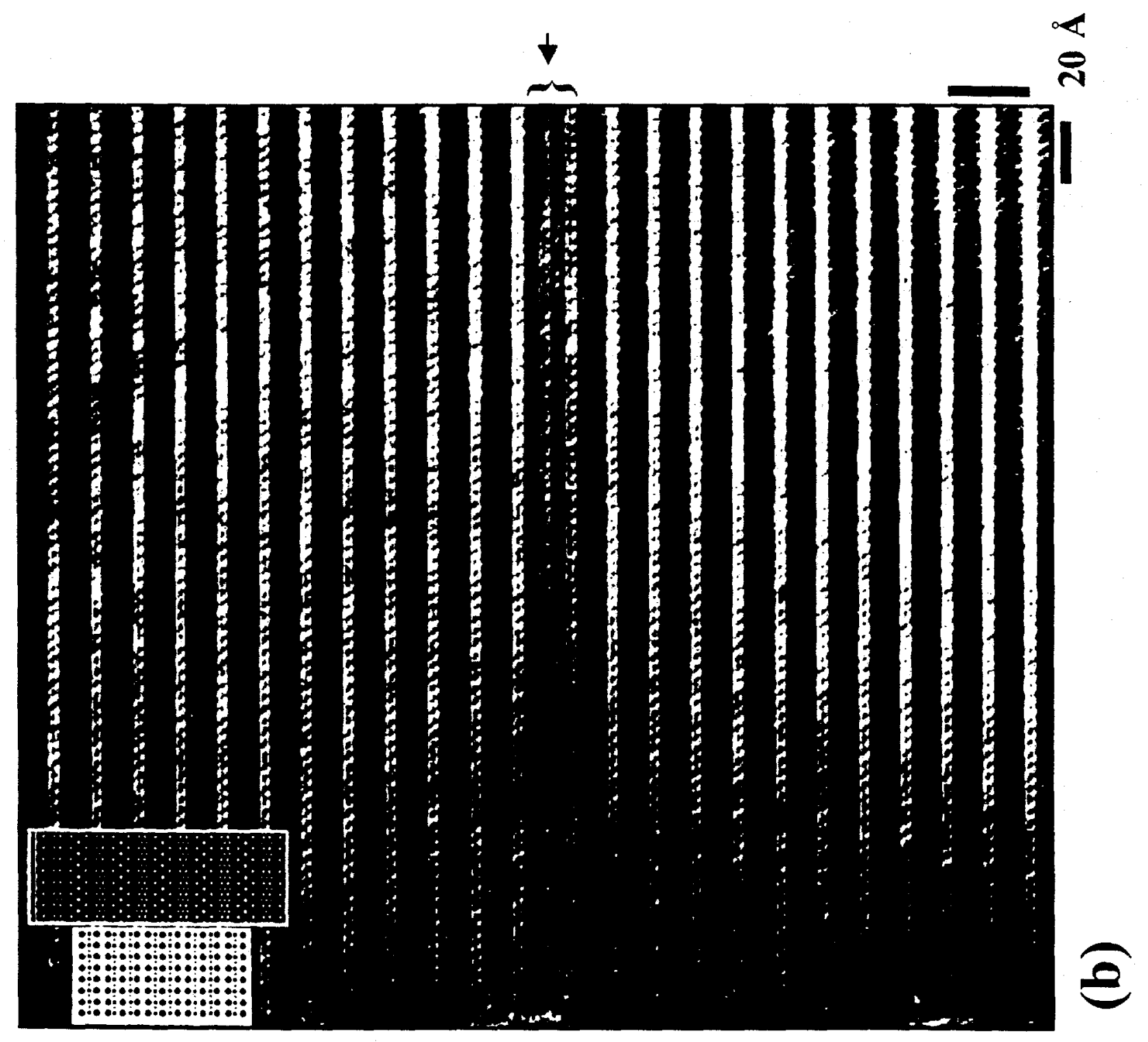

0
$\dot{1}$
0 

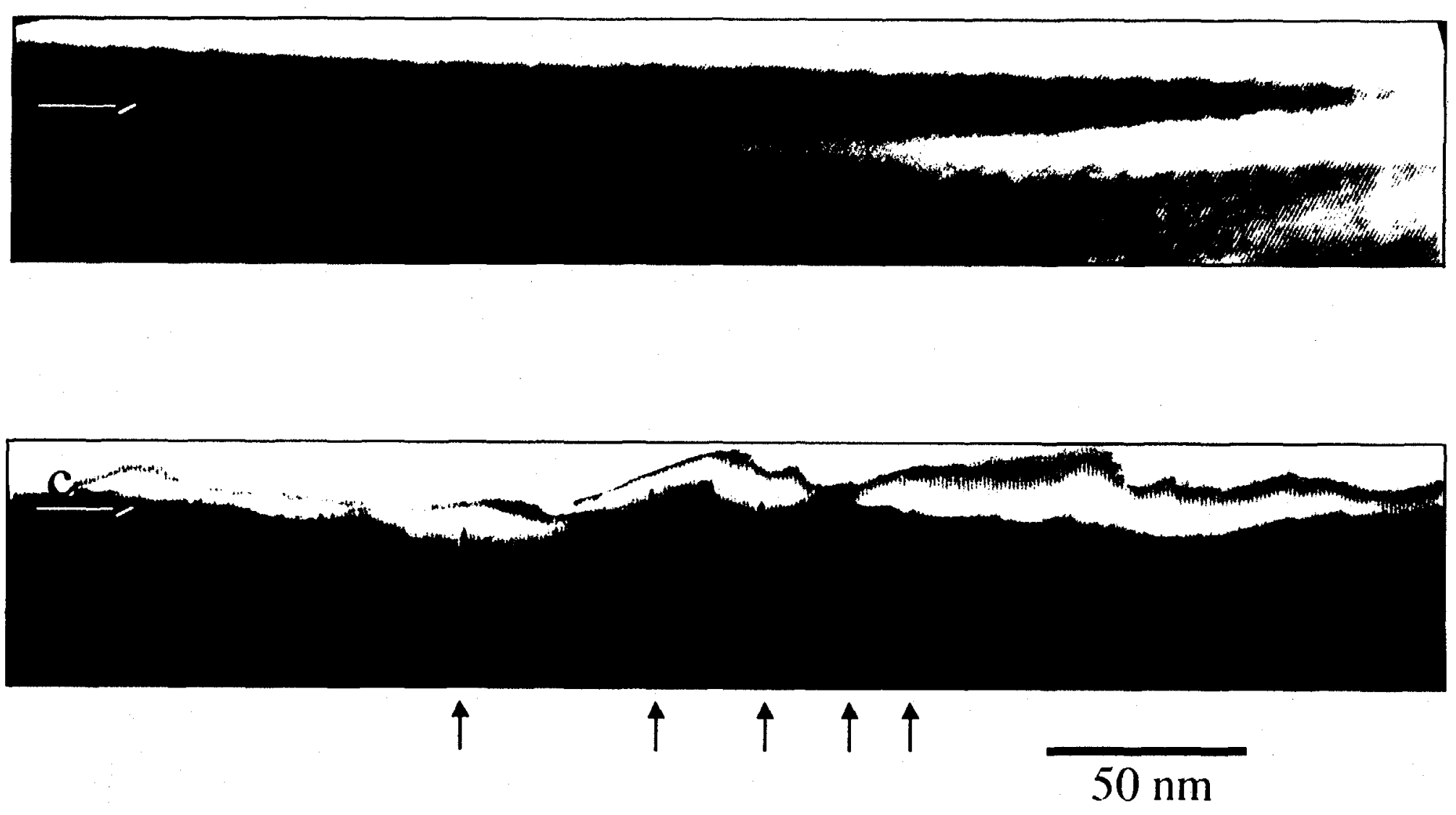

Fig. 3 


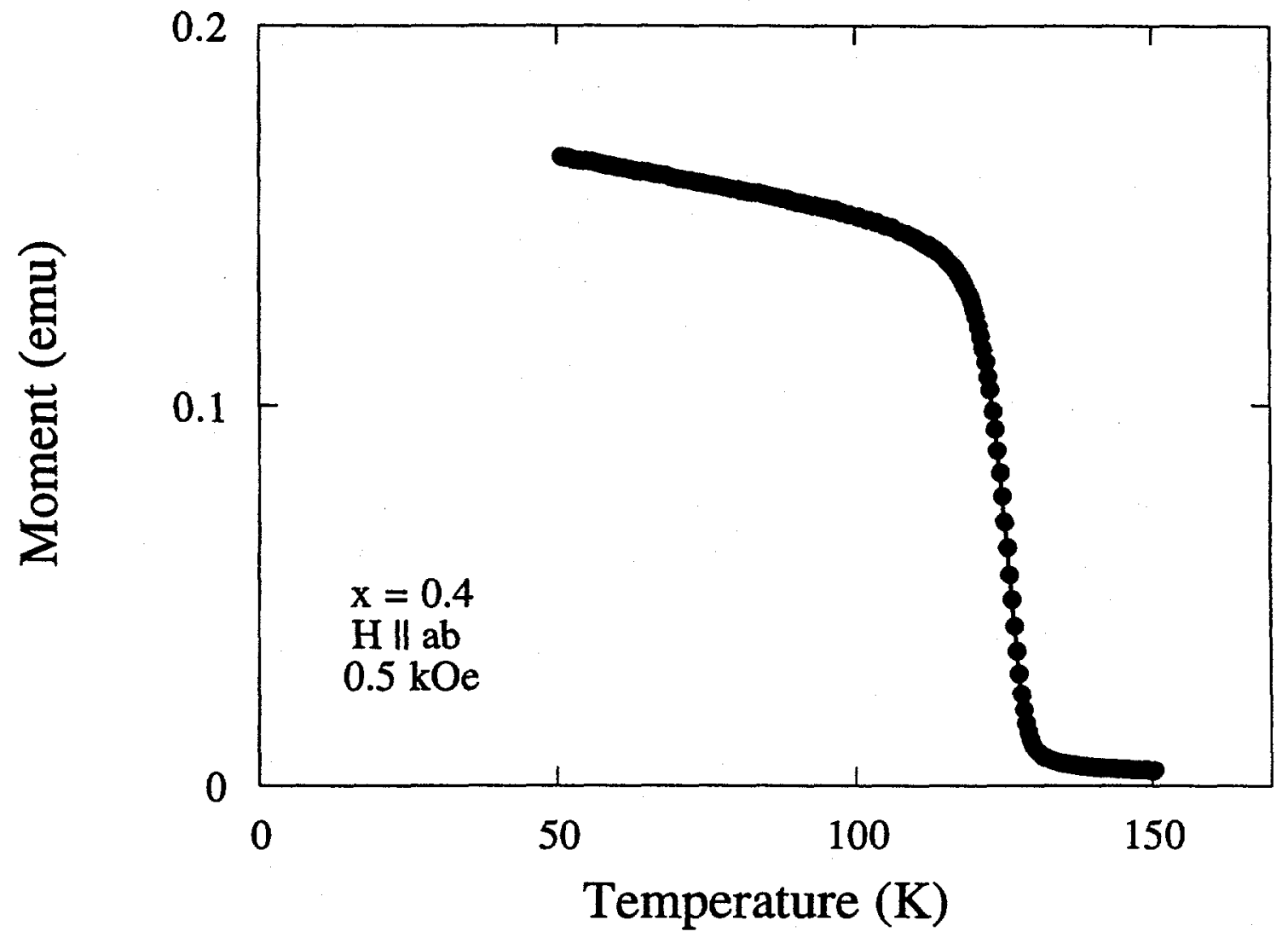

Fig. 4 

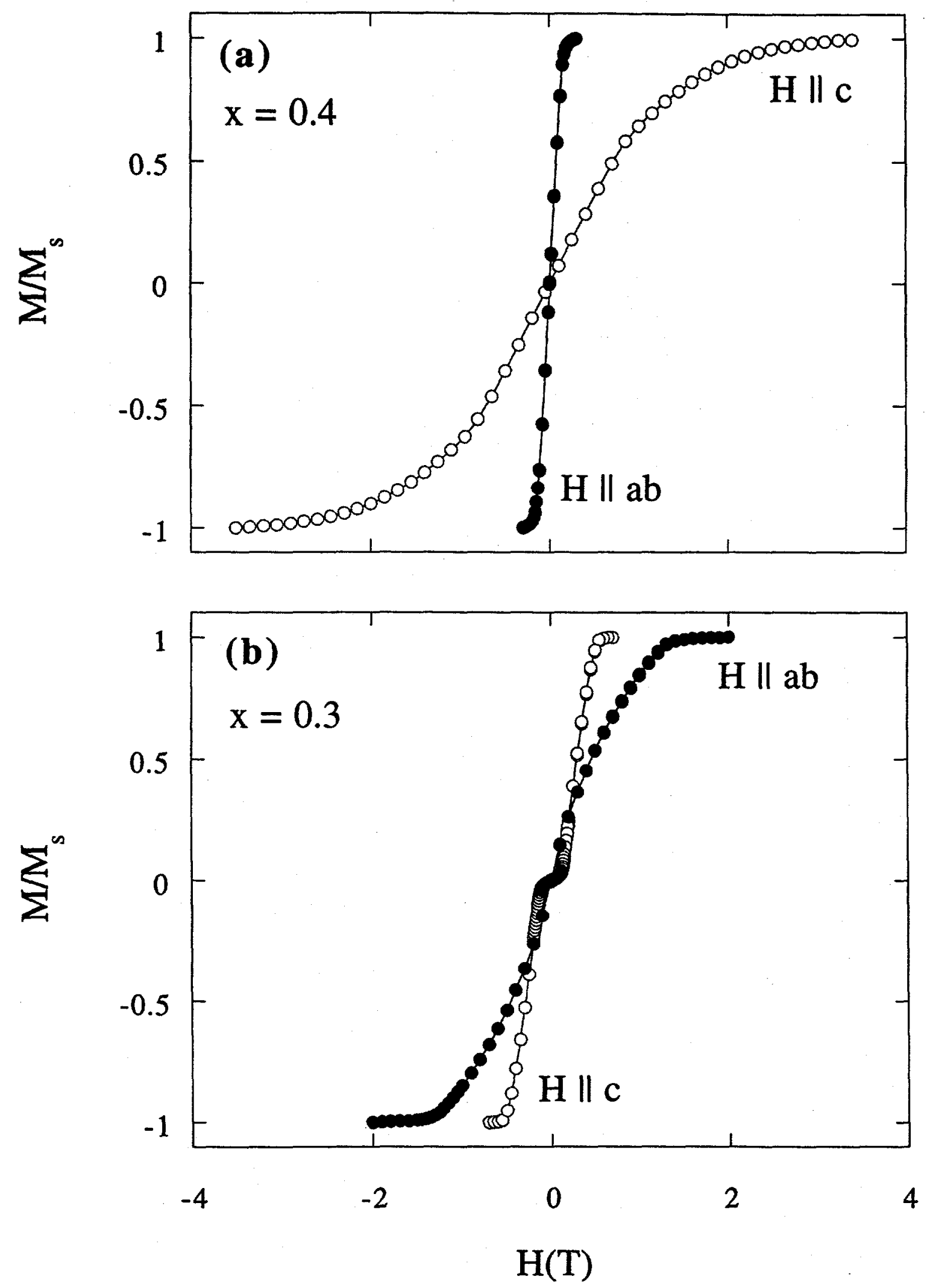

Fig. 5 


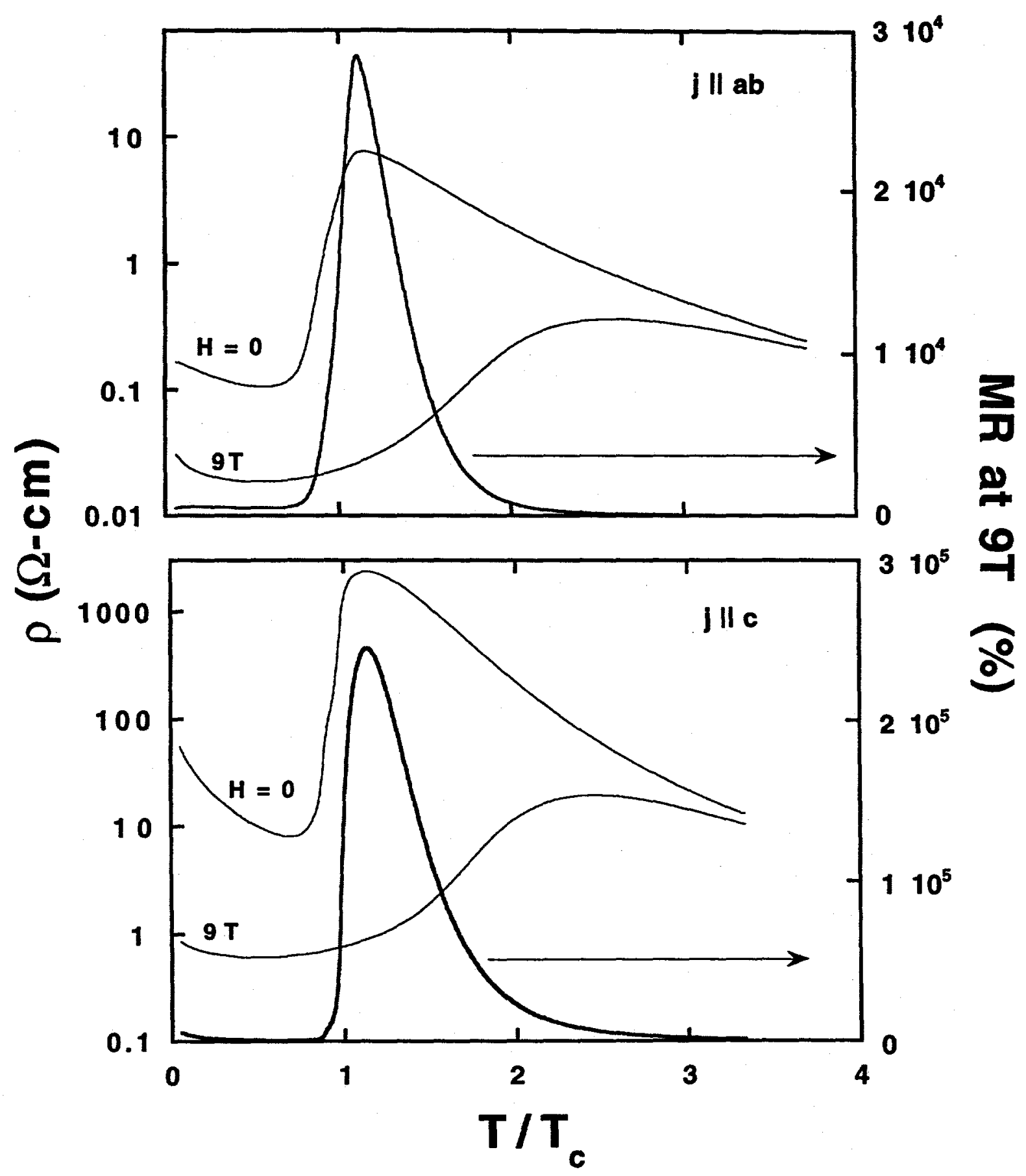

Fig.6 

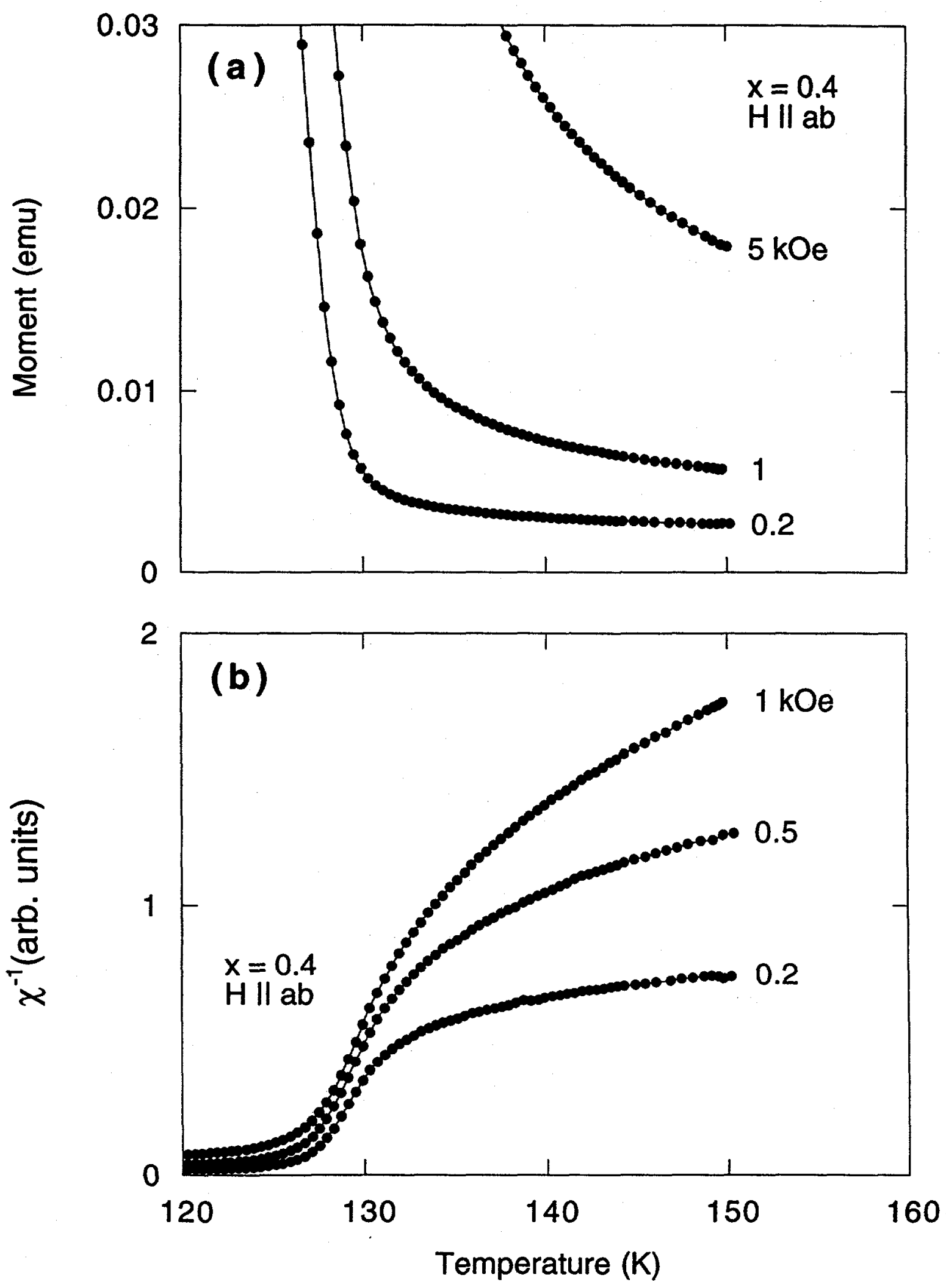

Fig. 7 


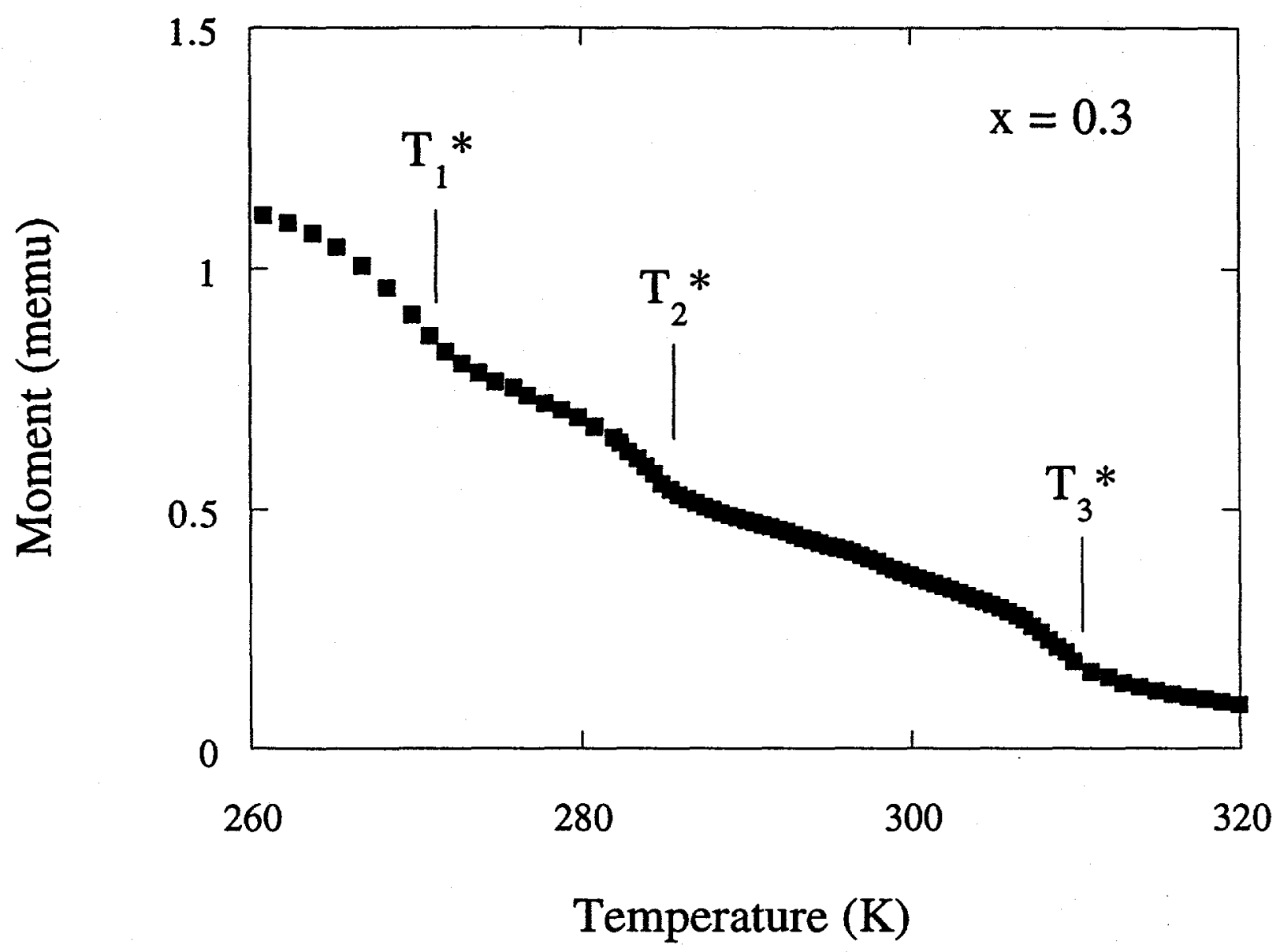

Fig. 8 


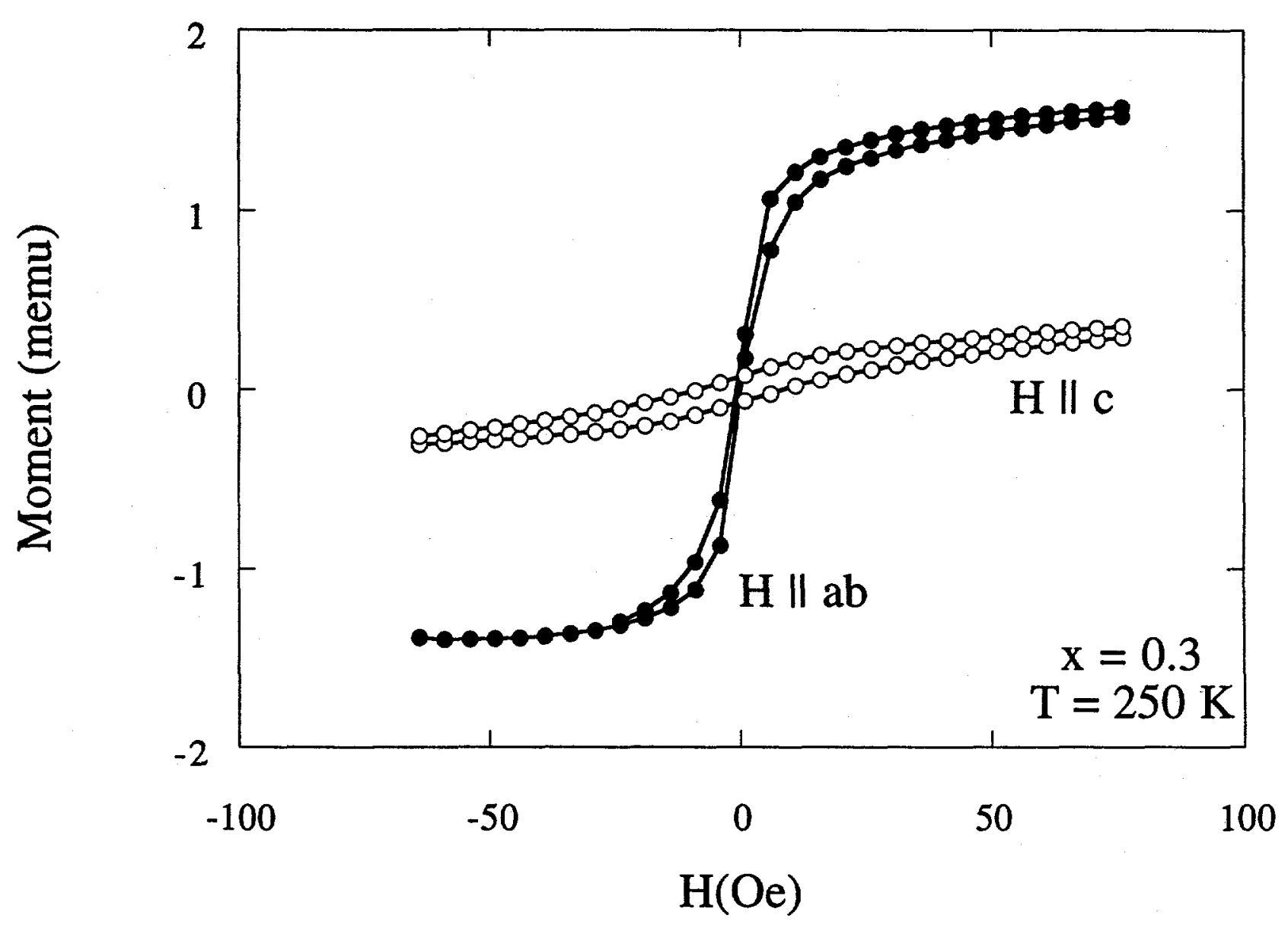

Fig. 9 